\title{
Psychedelics, psychosis and dreaming
}

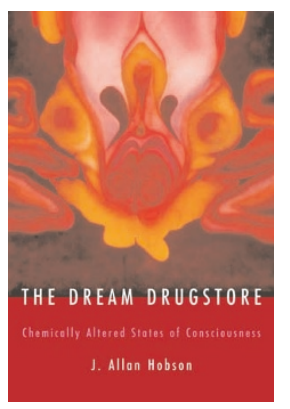

Drugs have long been the royal road to understanding brain function. Molecular aspects of depression were a total mystery until the advent of antidepressant drugs of which influences on serotonin and norepinephrine indicated a role for these molecules in mood regulation. Similarly, the first steps toward alleviating our ignorance of schizophrenia came with the discovery of the anti-psychotic neuroleptic drugs and their blockade of dopamine receptors. Psychedelic drugs have been a source of fascination to those trying to understand altered states of consciousness and perception. The term 'psychedelic' was coined by Humphrey Osmond from Greek words meaning 'enhanced consciousness,' and referred to the extraordinary changes in self-perception elicited by these drugs. From the first description in 1943 by Albert Hoffman of their extraordinary actions, some investigators have likened the psychotomimetic effects of such drugs to the mental aberrations of schizophrenia. Today, most psychiatrists feel that psychedelic drug psychosis is not a particularly meaningful drug model of schizophrenia, but they remain certain that the mental changes caused by these drugs may tell us something about the pathophysiology of psychosis. In his new book, Allan Hobson, one of America's most distinguished dream researchers, links dreaming, psychedelic states and psychosis.

Hobson's volume is an ambitious undertaking, covering topics ranging from psychological studies of consciousness, basic research on dreaming, and the pharmacology of many drugs, to

Solomon H. Snyder is in the Department of

Neuroscience, The Johns Hopkins University, 725 North Wolfe Street, Baltimore, Maryland 21205, USA.

e-mail:ssnyder@bs.jhmi.edu novel ways of treating mental illness. He begins by reviewing scientific models of consciousness, and he goes on to compare psychoanalytic formulations of dreaming with his own model, which is based on modern brain research. The psychoanalytic formulation holds that dreams are a mechanism of providing wish fulfillment, but that they do so in disguise. In contrast, Hobson presents the model that he and Robert McCarley developed more than twenty years ago, which shows that dreams are initiated by brain activation during sleep, with the visual imagery dependent on activation of the higher, integrative visual centers in the brain. The bizarre nature of dreams is attributed to loss of inhibitions that normally prevent the assembly of discordant elements in a visual scene. He shows how dreams recreate events that happened during the preceding day, and he clarifies many of the key differences between the waking and dreaming states.

The second half of the book is a brief textbook of psychopharmacology; it covers all the major psychoactive drugs in an eminently lucid presentation that focuses on their interactions with sleep mechanisms. Here, Hobson is not afraid to make strong value judgments that might not sit well with the pharmaceutical industry. For instance, he critically reviews influences of the 'safe' selective serotonin reuptake inhibitors (SSRIs). He shows how they disrupt distinctions between dreaming (REM) and nondreaming states, causing alterations in sleep architecture that can last for up to a year after drug discontinuation. $\mathrm{He}$ writes, "The SSRIs may well make us feel peppier in the daytime, but the cost is a blight on our sleep architecture that rivals the Russian bombardment of Grozny in Chechnya." Hobson is by no means self-important and freely admits errors of his own and other sleep researchers: "Basic sleep researchers make mistakes that are every bit as egregious and every bit as persistent as those of our clinical colleagues. From 1969 to 1975 it was widely held that serotonin promoted sleep. If that were true, than the SSRIs would be sedatives, not sleep saboteurs."

The heart of the book's conceptualizations come with a critical analysis of the mental effects of LSD, drawing heavily from the classic descriptions of Albert Hoffman himself. Hobson then reviews literature on the role of serotonin in psychedelic states and dreaming, because LSD is a molecular mimic of serotonin and, along with the other psychedelic drugs, is thought to act by binding to a subtype of serotonin receptor.

The book culminates with a synthetic approach suggesting implications for new types of psychotherapy. Its underpinnings are based on the lessons neurophysiology teaches us of the link between the conscious and unconscious mind, viewing the unconscious as a friend rather than an enemy. Practical advice consists of replacing the distancing 'neutrality' of the psychoanalyst with a warm, supportive relationship.

All in all, Hobson succeeds in providing a fresh perspective on the mental alterations that are common to dreaming, psychosis and psychedelic drug actions. The book is written in a lively style with complex neurophysiologic and pharmacologic analyses made lucid enough for any intelligent lay reader. It is one of those rare books that will be of importance to the most sophisticated researchers and clinical practitioners and yet an accessible and a fascinating read for many non-specialists. 